\title{
NONEMPTY INTERSECTION THEOREMS MINIMAX THEOREMS WITH APPLICATIONS IN GENERALIZED INTERVAL SPACES
}

\author{
DA-CHENG WANG
}

(Received 7 August 2000)

\begin{abstract}
We establish some new topological types of nonempty intersection theorems in more general topological spaces without linear structure. As applications, we utilize results to study the minimax problems, coincidence problems, and economy equilibrium problems in generalized interval spaces and some new results are obtained.
\end{abstract}

2000 Mathematics Subject Classification. 49K35, 54D20, 54D05, 54H25.

1. Introduction and preliminaries. Recently, some versions of Fan's KKM theorem and Fan's minimax theorem in topological spaces without linear structure have been considered by Cheng and Lin [2], Lin et al. [10, 11], and Tian [14]. The purpose of this paper is first to introduce and establish a class of more general topological spacegeneralized interval space, which has no linear structure and contains $H$-space, interval space as its special cases, and so as it contains strong interval space [2], convex space, contractible space, and topological vector space as its special cases. With the aid of this kind of topological frameworks some new topological types of nonempty intersection theorems are established in Section 2. As applications, we utilize these results to obtain some minimax theorems and coincidence theorems in generalized interval space. The results presented in this paper not only contain many recent results of Stacho [13], Kindler and Trost [8], Cheng and Lin [2], Brezis et al. [1], Tuy [7], Komornik [9], Geraghty and Lin [4], Sion [12], Wu [15], Hartung [6], and the famous Neumann's saddle theorem as its special cases, but also improve and extend the corresponding results of $[1,2,4,5,6,7,8,9,10,11,12,14]$.

For the sake of convenience, we first give some definitions and notations.

Let $X$ and $Y$ be two Hausdorff topological spaces. We denote by $C(X, Y)$ the set of all continuous mappings from $X$ to $Y$. Let $L B$ be a subset of $X$, we denote by $\operatorname{cl} B$

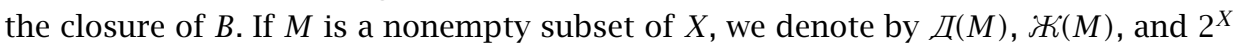
the families of all nonempty finite subsets, all nonempty connected subsets, and all subsets of $M$, respectively. We denote $\mathbb{R}=(-\infty,+\infty)$.

DEFINITION 1.1 (see [2]). Let $E$ be a linear ordered space. We call $E$ a completely dense linear space, if each subset of $E$ has a least upper bound and for any $Z_{1}, Z_{2} \in E$ with $Z_{1}<Z_{2}$, there exists $z \in E$ such that $Z_{1}<z<Z_{2}$.

Definition 1.2. Let $X$ be a topological space, $X$ is called a generalized interval space, if there exists a mapping $\Gamma: X \times X \rightarrow \mathscr{W}(X)$ such that for any $\left(\mathscr{X}_{1}, \mathscr{X}_{2}\right) \in X \times X$, 
$\Gamma\left(\mathscr{X}_{1}, \mathscr{X}_{2}\right)=\Gamma\left(\mathscr{X}_{2}, \mathscr{X}_{1}\right)$, and $\Gamma\left(\mathscr{X}_{1}, \mathscr{X}_{2}\right)$ is called a generalized interval associated with $\mathscr{X}_{1}, \mathscr{X}_{2}$.

DeFinITION 1.3. Let $X$ be a generalized interval space, $Y$ a topological space, and $E$ a completely dense linear space. A subset $B$ of $X$ is called $T$-convex set, if for any $\mathscr{X}_{1}, \mathscr{X}_{2} \in B$, we have $\Gamma\left(\mathscr{X}_{1}, \mathscr{X}_{2}\right) \subset B$. A mapping $f: X \rightarrow E$ is called $T$-quasi-concave, if for any $\mathscr{X}_{1}, \mathscr{X}_{2} \in X$, there exist $\mathscr{Y}_{1}, \mathscr{Y}_{2} \in \Gamma\left(\mathscr{X}_{1}, \mathscr{X}_{2}\right)$ such that $f\left(\mathscr{y}_{i}\right) \geq f\left(\mathscr{X}_{i}\right), i=1,2$, and for any $z \in E$, the set $\{x \in X: f(x) \geq z\}$ is $T$-convex. We call $F: X \rightarrow 2^{Y}$ a $T$-KKM mapping, if for any $\mathscr{X}_{1}, \mathscr{X}_{2} \in X$ we have $F(x) \subset F\left(\mathscr{X}_{1}\right) \cup F\left(\mathscr{X}_{2}\right)$ for all $x \in \Gamma\left(\mathscr{X}_{1}, \mathscr{X}_{2}\right)$, and there exist $\mathscr{Y}_{1}, \mathscr{Y}_{2} \in \Gamma\left(\mathscr{X}_{1}, \mathscr{X}_{2}\right)$ such that $F\left(\mathscr{Y}_{i}\right) \subset F\left(\mathscr{X}_{i}\right), i=1,2$.

REMARK 1.4. For Definitions 1.2 and 1.3 it is easy to see that both interval space and $H$-space are the special cases of generalized interval space, and so as all the strong interval space [2], convex space, contractible space, and topological vector space are the special cases of generalized interval space. For $H$-space, the $H$-convex set is $T$ convex. For topological linear space, a convex subset is $T$-convex, a quasi-concave function is $T$-quasi-concave.

REMARK 1.5. It is obvious that a nonempty $T$-convex subset in a generalized interval space is also a generalized interval space.

DEFINITION 1.6 (see [14]). Let $X$ and $Y$ be two topological spaces. A mapping $F: X \rightarrow 2^{Y}$ is called transfer closed valued, if for each $x \in X, y \notin F(x)$ implies that there exists a point $\mathscr{X} \bullet X$ such that $\mathrm{y} \notin \mathrm{cl} F(\mathscr{X} \bullet)$.

LEMмA 1.7. Let $X$ be a generalized interval space, $E$ a completely dense linear ordered space, and $f: X \rightarrow E$ a mapping. Then $f$ is T-quasi-concave if and only if for any $\mathscr{X}_{1}, \mathscr{X}_{2} \in X$, there exist $\mathscr{Y}_{1}, \mathscr{Y}_{2} \in \Gamma\left(\mathscr{X}_{1}, \mathscr{X}_{2}\right)$ such that $f\left(\mathscr{Y}_{i}\right) \geq f\left(\mathscr{X}_{i}\right), i=1,2$, and $f(x) \geq \min \left\{f\left(\mathscr{X}_{1}\right), f\left(\mathscr{X}_{2}\right)\right\}$ for all $x \in \Gamma\left(\mathscr{X}_{1}, \mathscr{X}_{2}\right)$.

Proof. The proof follows from Definition 1.3 immediately.

LEMMA 1.8. Let $X$ be a generalized interval space, $Y$ a topological spaces, and $E$ a completely dense linear ordered space. Let $\alpha \in E$ and $\varphi: X \times X \rightarrow E$ be T-quasi-concave in $X$. If we define a mapping $F: X \rightarrow 2^{Y}$ by $F(x)=\{y \in Y: \varphi(x, y) \leq \alpha\}$ for all $x \in X$, then $F$ is a T-KKM mapping.

Proof. Since $\varphi$ is $T$-quasi-concave in $X$, for any given $\mathscr{X}_{1}, \mathscr{X}_{2} \in X$ and for any $x \in \Gamma\left(\mathscr{X}_{1}, \mathscr{X}_{2}\right)$ we have $\varphi(x, y) \geq \min \left\{\varphi\left(\mathscr{X}_{1}, y\right), \varphi\left(\mathscr{X}_{2}, y\right)\right\}$ for any $y \in Y$, and there exist $\mathscr{X}_{1}{ }^{\bullet}, \mathscr{X}_{2}{ }^{\bullet} \in \Gamma\left(\mathscr{X}_{1}, \mathscr{X}_{2}\right)$ such that $\varphi\left(\mathscr{X}_{i} \cdot y\right) \geq \varphi\left(\mathscr{X}_{i}, y\right), i=1,2$ for all $y \in Y$. Hence $F\left(\mathscr{X}_{i}^{\bullet}\right) \subset F\left(\mathscr{X}_{i}\right), i=1$, 2, for all $x \in \Gamma\left(\mathscr{X}_{1}, \mathscr{X}_{2}\right)$, we have $F(x) \subset \bigcup_{i=1}^{2} F\left(\mathscr{X}_{i}\right)$. This implies that $F$ is a $T$-KKM mapping. Let $X, Y$ be two topological spaces, $F: X \rightarrow 2^{Y}$. In the sequel we denote $\operatorname{cl}\{F(x)\}=\operatorname{cl} F(x)$.

\section{Some nonempty intersection theorems}

THEOREM 2.1. Let $X$ be a generalized interval space, $Y$ a topological space, and $F: X \rightarrow 2^{Y}$ an upper semi-continuous T-KKM mapping. Suppose that the following conditions are satisfied:

(1) for any $x \in X, F(x)$ is a nonempty closed (open) set in $Y$; 
(2) for any $A \in Д(X), \bigcap_{x \in A} F(x) \in \mathscr{W}(Y)$,

then

(1) $\{F(x): x \in X\}$ has the finite intersection property;

(2) if there exists an $A_{0} \in Д(X)$ such that $\bigcap_{x \in A} \operatorname{cl} F(x)$ is compact, then

$$
\bigcap_{x \in A} \operatorname{cl} F(x) \neq \varnothing .
$$

Proof. Letting $\Omega=\{F(x): x \in X\}$, and by using induction we prove that $\Omega$ has the finite intersection property.

By condition (1), for any $F(x)$ in $\Omega, x \in X$ is nonempty. Suppose that for any $n$ elements in $\Omega, n \geq 2$, their intersection is nonempty, next we prove that for any $n+1$ elements in $\Omega$, their intersection is also nonempty. Suppose the contrary, then there exist $\mathscr{X}_{1}, \mathscr{X}_{2}, \ldots, \mathscr{X}_{n+1} \in X$ such that $\bigcap_{i=1}^{n+1} F\left(\mathscr{X}_{i}\right)=\varnothing$. Letting $H=\bigcap_{i=1}^{n+1} F\left(\mathscr{X}_{i}\right)$, then

$$
\bigcap_{i=1}^{2} H \cap F\left(\mathscr{X}_{i}\right)=\varnothing \text {. }
$$

By condition (2) and the assumption of induction, for any $x \in X$

$$
H \cap F(x) \text { is nonempty and connected. }
$$

Since $F$ is a $T$-KKM mapping, $F(x) \subset \bigcup_{i=1}^{2} F\left(\mathscr{X}_{i}\right)$ for all $x \in \Gamma\left(\mathscr{X}_{1}, \mathscr{X}_{2}\right)$ and there exist $\mathscr{X}_{1} \cdot \mathscr{X}_{2} \cdot \in \Gamma\left(\mathscr{X}_{1}, \mathscr{X}_{2}\right)$ such that $F\left(\mathscr{X}_{i}^{\bullet}\right) \subset F\left(\mathscr{X}_{i}\right), i=1,2$. Hence,

$$
H \cap F(x) \subset \bigcup_{i=1}^{2} H \cap F\left(\mathscr{X}_{i}\right), \quad \forall x \in \Gamma\left(\mathscr{X}_{1}, \mathscr{X}_{2}\right),
$$

letting $D_{j}=\left\{x \in \Gamma\left(\mathscr{X}_{1}, \mathscr{X}_{2}\right): H \cap F(x) \subset H \cap F\left(\mathscr{X}_{i}\right)\right\}, j=1,2$, then $\mathscr{X}_{i} \bullet \in D_{i}, i=1,2$. It follows from (2.2), (2.3), (2.4), and condition (1) that $D_{1} \cup D_{2}=\Gamma\left(\mathscr{X}_{1}, \mathscr{X}_{2}\right)$. Since $\Gamma\left(\mathscr{X}_{1}, \mathscr{X}_{2}\right)$ is connected, we know that either $\operatorname{cl} D_{1} \cap D_{2}$ or $\operatorname{cl} D_{2} \cap D_{1}$ is nonempty. Without loss of generality, we can assume $\operatorname{cl} D_{2} \cap D_{1} \neq \varnothing$. Hence there exists an $x_{0} \in \operatorname{cl} D_{2} \cap D_{1}$. By (2.2), we have $\left(H \cap F\left(\mathscr{X}_{0}\right)\right) \cap\left(H \cap F\left(\mathscr{X}_{2}\right)\right)=\varnothing$, and it is easy to prove that there exists an open set $U$ containing $F\left(\mathscr{X}_{0}\right)$ such that $(H \cap U) \cap\left(H \cap F\left(\mathscr{X}_{2}\right)\right)=\varnothing$. In fact, if $F$ is open valued, it is sufficient to take $U=F\left(\mathscr{Q}_{0}\right)$; if $F$ is closed valued, taking $U=Y \backslash\left(H \cap F\left(\mathscr{C}_{2}\right)\right)$, then $U$ is an open set in $Y$ and

$$
(H \cap U) \cap\left(H \cap F\left(\mathscr{X}_{2}\right)\right)=\varnothing, \quad U \supset\left(H \cap F\left(\mathscr{X}_{0}\right)\right) \neq \varnothing .
$$

Moreover, for any $y \notin U$, then $y \in H \cap F\left(\mathscr{X}_{2}\right)$ and $y \notin H \cap F\left(\mathscr{L}_{0}\right)$, and so $y \notin F\left(\mathscr{L}_{0}\right)$. Hence $F\left(\mathscr{X}_{0}\right) \subset U$. This means that $U$ satisfies the desired condition.

By the upper semi-continuity of $F$, there exists an open neighborhood $V$ of $\mathscr{X}_{0}$ such that for any $x \in V, F(x) \subset U$. Since $\mathscr{X}_{0} \in \operatorname{cl} D_{2}, V \cap D_{2} \neq \varnothing$. Taking $\mathscr{x} \bullet \in \cap \cap D_{2}$, we have $H \cap F\left(\mathscr{X}^{\bullet}\right) \subset H \cap F\left(\mathscr{C}_{2}\right), H \cap F(\mathscr{X} \bullet) \subset H \cap U$. Hence we have $\left(H \cap F\left(\mathscr{X}_{2}\right)\right)(H \cap U) \supset$ $H \cap F(\mathscr{Q} \bullet) \neq \varnothing$. This contradicts (2.5), therefore $\Omega$ has the finite intersection property.

In addition, if there exists an $A_{0} \in Д(M)$ such that $\bigcap_{x \in A_{0}} \operatorname{cl} F(x)$ is compact. Letting $K=\bigcap_{x \in A_{0}} \operatorname{cl} F(x)$, it follows from the finite intersection property of $\Omega$ and $A_{0}$ being finite that $\{\operatorname{cl} F(x) \cap K: x \in X\}$ is a family of closed set in the compact set $K$ and has the finite intersection property, therefore $\bigcap_{x \in X}(\operatorname{cl} F(x) \cap K)=\bigcap_{x \in X} \operatorname{cl} F(x) \neq \varnothing$. This completes the proof. 
TheOREM 2.2. Let $X$ be a generalized interval space, $Y$ a topological space, $F: X \rightarrow 2^{Y}$ a mapping with nonempty values and satisfy the following conditions:

(1) $F$ is transfer closed valued;

(2) $\operatorname{cl} F: X \rightarrow 2^{Y}$ is an upper semi-continuous T-KKM mapping;

(3) for each $A \in Д(X), \bigcap_{x \in X} \operatorname{cl} F(x) \in \Psi(Y)$;

(4) there exists an $A_{0} \in Д(X)$ such that $\bigcap_{x \in A_{0}} \operatorname{cl} F(x)$ is compact, then $\bigcap_{x \in X} F(x) \neq \varnothing$.

Proof. First we prove $\bigcap_{x \in X} F(x)=\bigcap_{x \in X} \mathrm{cl} F(x)$. In fact, since $\bigcap_{x \in X} F(x) \subset$ $\bigcap_{x \in X} \operatorname{cl} F(x)$, it is sufficient to prove that $\bigcap_{x \in X} F(x) \supset \bigcap_{x \in X} \operatorname{cl} F(x)$. Suppose the contrary, then there exists $Y_{0} \in \bigcap_{x \in X} \operatorname{cl} F(x)$ such that $y_{0} \notin \bigcap_{x \in X} F(x)$. Hence there exists an $x_{0} \in X$ such that $y_{0} \notin F(x)$. By condition (1), there exists $x^{\prime} \in X$ such that $y_{0} \notin \mathrm{cl} F(x)$. This contradicts the choice $y_{0}$. Hence the desired conclusion is proved.

It is obvious that $\mathrm{cl} F: X \rightarrow 2^{Y}$ satisfies all the conditions in Theorem 2.1. By Theorem 2.1, we know that $\bigcap_{x \in X} F(x)=\bigcap_{x \in X} \operatorname{cl} F(x) \neq \varnothing$.

THEOREM 2.3. Let $X$ be a generalized interval space, $Y$ a topological space, $S \in$ $C(X, Y)$ a given mapping, and $F: X \rightarrow 2^{Y}$ a mapping satisfying the following conditions:

(1) $F$ is transfer closed valued;

(2) $\operatorname{cl} F: X \rightarrow 2^{Y}$ is an upper semi-continuous T-KKM mapping;

(3) there exists a compact set $L$ in $Y$ and a subset $K$ in $X$ such that for any $A \in Д(X)$ there exists a compact $T$-convex subset $D \subset X$ with $K \bigcup A \subset D$ such that

(a) for any $x \in D, \operatorname{cl} F(x) \cap s(D) \neq \varnothing$;

(b) for any finite set $\left\{x_{1}, x_{2}, \ldots, x_{n}\right\} \subset D, \bigcap_{i=1}^{n}\left(\operatorname{cl} F\left(x_{i}\right) \cap s(D)\right) \in \nVdash(Y)$;

(c) $\bigcap_{x \in D}(\operatorname{cl} F(x) \cap s(D)) \subset L$, then $\bigcap_{x \in X} F(x) \neq \varnothing$.

Proof. By condition (1), $\bigcap_{x \in X} F(x)=\bigcap_{x \in X} \mathrm{cl} F(x)$. Hence in order to prove the conclusion of the theorem, it suffices to prove that $\bigcap_{x \in X} \operatorname{cl} F(x) \neq \varnothing$. Since $L$ is compact, for the purpose, it suffices to prove that $\{\operatorname{cl} F(x) \cap L: x \in X\}$ has the finite intersection property. For any $A \in Д(X)$, let $D$ be the set satisfying condition (3). Hence we have

$$
\bigcap_{x \in A}(\operatorname{cl} F(x) \cap L) \supset \bigcap_{x \in D}(\operatorname{cl} F(x) \cap s(D)) .
$$

Letting $Y_{0}=s(D)$, by the continuity of $s$ and compactness of $D$, we know that $Y_{0}$ is a compact subset of $Y$.

Next we prove that $\bigcap_{x \in D}\left(\operatorname{cl} F(x) \cap Y_{0}\right) \neq \varnothing$. In fact, letting $G(x)=\operatorname{cl} F(x) \cap Y_{0}$, $x \in D$, by condition (3)(a), for any $x \in X, G(x)$ is nonempty compact. Since $D$ is $T$ convex, $D$ is a generalized interval space. It is easy to prove that $G: D \rightarrow 2^{Y}$ satisfies all conditions in Theorem 2.1. By Theorem 2.1, $\{G(x): x \in X\}$ has the finite intersection property. Therefore, we have $\bigcap_{x \in D} G(x) \neq \varnothing$, that is, $\bigcap_{x \in D}\left(\operatorname{cl} F(x) \cap Y_{0}\right) \neq \varnothing$. By (2.6), $\bigcap_{x \in A}(\operatorname{cl} F(x) \cap L) \neq \varnothing$. This implies that $\{\operatorname{cl} F(x) \cap L: x \in X\}$ has the finite intersection property.

THEOREM 2.4. Let $X$ be a generalized interval space, $Y$ a compact topological space, and $G: X \rightarrow 2^{Y}$ a mapping with nonempty values and satisfy the following conditions: 
(1) $G$ is transfer closed valued;

(2) $\operatorname{cl} G: X \rightarrow 2^{Y}$ is a T-KKM mapping;

(3) for any $A \in Д(X), \bigcap_{x \in A} \operatorname{cl} G(x) \in \mathscr{W}(Y)$;

(4) $\mathrm{cl} G$ is closed graph,

then $\bigcap_{x \in X} G(x) \neq \varnothing$.

Proof. Since $\mathrm{cl} G$ is a closed graph and $Y$ is compact, by using Fan [3, Lemma 2] we know that $\operatorname{cl} G$ is upper semi-continuous. The conclusion follows from Theorem 2.2 immediately.

3. Applications to minimax problems. As applications, we use the results obtained in Section 2 to give some minimax theorems in generalized interval spaces.

THEOREM 3.1. Let $X$ be a generalized interval space, $Y$ a compact topological space, $E$ a completely dense linear ordered space, and $\varphi: X \times X \rightarrow E$ a lower semi-continuous function satisfying the following conditions:

(1) $\varphi(x, y)$ is $T$-quasi-concave in $x$;

(2) for any $A \in Д(X)$ and for any $\alpha \in E, \bigcap_{x \in A}\{y \in Y: \varphi(x, y) \leq \alpha\} \in \mathscr{W}(Y)$, then $\alpha_{\bullet}=\sup _{x \in X} \inf _{y \in Y} \varphi(x, y)=\inf _{y \in Y} \sup _{x \in X} \varphi(x, y)=\alpha^{\bullet}$.

Proof. It is obvious that $\alpha_{\bullet} \leq \alpha^{\bullet}$.

Now we prove that $\alpha_{\bullet} \geq \alpha^{\bullet}$. Without loss of generality, we can assume that $\alpha_{\bullet}$ is not the greatest element in $E$. For any $x \in X$ and for any given $\alpha>\alpha_{\bullet}$, let

$$
G(x, \alpha)=\{y \in Y: \varphi(x, y) \leq \alpha\} .
$$

Next we prove that $\left\{G(x, \alpha): x \in X, \alpha>\alpha_{0}\right\}$ has the finite intersection property. In fact, for any $n$ points $x_{1}, \ldots, x_{n} \in X$ and any $n$ elements $\alpha_{1}, \ldots, \alpha_{n} \in E$ with $\alpha_{i}>\alpha_{\text {. }}$, $i=1, \ldots, n$, since $E$ is a linear ordered space, we can assume that $\alpha_{n} \geq \cdots \geq \alpha_{1}>\alpha_{\text {. }}$. By the density of $E$, there exists an $\bar{\alpha} \in E$ such that $\alpha_{1}>\bar{\alpha}>\alpha_{\text {. }}$. Letting

$$
F(x)=G(x, \bar{\alpha}), \quad x \in X,
$$

by the lower semi-continuity of $\varphi$ and the choice of $\bar{\alpha} \in E$, we know that $F: X \rightarrow 2^{Y}$ is nonempty closed valued. This implies that $F$ satisfies condition (1) in Theorem 2.1. From condition (2), $F$ satisfies condition (2) in Theorem 2.1. In view of condition (1) and Lemma 1.7, we know that $F$ is a $T$-KKM mapping. In addition, since $\varphi: X \times Y \rightarrow E$ is lower semi-continuous, $F$ is closed graph. By Fan [3, Lemma 2], $F$ is upper semicontinuous. Therefore $F$ satisfies all the conditions in Theorem 2.1. By Theorem 2.1, $\{F(x): x \in X\}$ has the finite intersection property, and so $\bigcap_{i=1}^{n} F\left(x_{i}\right) \neq \varnothing$. Since $\bigcap_{i=1}^{n} F\left(x_{i}\right) \subset \bigcap_{i=1}^{n} G\left(x_{i}, \alpha_{i}\right),\left\{G(x, \alpha): x \in X, \alpha>\alpha_{0}\right\}$ has the finite intersection property.

On the other hand, for any $x \in X$ and for any $\alpha>\alpha_{\bullet}, G(x, \alpha)$ is closed. Since $Y$ is compact, $\bigcap_{x \in X, \alpha>\alpha} G(x, \alpha) \neq \varnothing$. Hence, there exists $\bar{y} G(x, \alpha)$ for all $x \in X$ and for all $\alpha>\alpha_{\text {e }}$, that is, $\varphi(x, \bar{y}) \leq \alpha$ for all $x \in X$ and for all $\alpha>\alpha_{\text {. }}$. Hence, we have $\inf _{y \in Y} \sup _{x \in X} \varphi(x, y) \leq \alpha$, for all $\alpha>\alpha$.

Since $E$ is a dense linear ordered space, we know that $\alpha^{\bullet}=\inf _{y \in Y} \sup _{x \in X} \varphi(x, y) \leq \alpha$. Summing up the above arguments, we have $\alpha_{\bullet}=\alpha^{\bullet}$. This completes the proof. 
REMARK 3.2. Theorem 3.1 not only contains Theorem 1 in Shacho [13] as its special case but also relaxes its continuous condition.

THEOREM 3.3. Let $X$ be a generalized interval space, $Y$ a topological space, $E$ a completely dense linear ordered space, and $\varphi, \phi: X \times X \rightarrow E$ two functions satisfying the following conditions:

(1) $\varphi(x, y) \leq \phi(x, y)$ for all $(x, y) \in X \times Y$;

(2) $\varphi(x, y)$ is $T$-quasi-concave in $x$, and it is upper semi-continuous on any generalized interval of $X$;

(3) $\varphi(x, y)$ is lower semi-continuous in $y$ and for any $A \in Д(X)$, the set $\bigcap_{x \in A}\{y \in$ $Y: \varphi(x, y)<\alpha\}$ is connected for all $\alpha \in E$

(4) there exists an $A_{0} \in Д(X)$ and $a z_{0}>\sup _{x \in X} \inf _{y \in Y} \phi(x, y)$ such that $\bigcap_{x \in A_{0}}\{y \in$ $\left.Y: \varphi(x, y) \leq z_{0}\right\}$ is compact,

then $\inf _{y \in Y} \sup _{x \in X} \varphi(x, y) \leq \sup _{x \in X} \inf _{y \in Y} \phi(x, y)$.

Proof. Letting $z_{\bullet}=\sup _{x \in X} \inf _{y \in Y} \phi(x, y)$, by the completeness of $E$, we know that $z$. exists. From condition (4), $z$. is not a largest element in $E$.

For any $x \in X$ and for any $z>z$, let $F(x, z)=\{y \in Y: \varphi(x, y) \leq z\}$. It follows from conditions (1) and (3) that $F(x, z)$ is a nonempty closed set. By condition (4), $\bigcap_{x \in A} F(x, z)$ is compact. Denote $\Omega=\{F(x, z): x \in X, z \in Z\}$. Next we prove that $\Omega$ has the finite intersection property. In fact, we have proved that each element in $\Omega$ is nonempty. Suppose for any $n$ elements in $\Omega$ their intersection is nonempty. Now we prove that any $n+1$ elements in $\Omega$ their intersection is nonempty. Suppose the contrary, then there exist $\mathscr{X}_{1}, \ldots, \mathscr{X}_{n}, \mathscr{X}_{n+1}$ in $X$ and $z_{1}, \ldots, z_{n}, z_{n+1}$ in $E$ with $z_{i}>z_{\text {., }}$ $i=1,2, \ldots, n+1$ such that $\bigcap_{i=1}^{n+1} F\left(x_{i}, z_{i}\right)=\varnothing$. Without loss of generality, we can assume that $z_{0}<z_{1} \leq \cdots \leq z_{n} \leq z_{n+1}$. By the density of $E$, there exists a $\bar{z} \in E$ such that $z_{1}>\bar{z}>z_{\text {. . Letting }}$

$$
\begin{gathered}
T(x, z)=\{y \in Y: \varphi(x, y)<z\}, \quad \forall x \in X, \forall z>z_{\bullet} ; \\
T(x)=T\left(x, z_{1}\right), \quad \forall x \in X ; H=\bigcap_{i=3}^{n+1} T\left(x_{i}\right) .
\end{gathered}
$$

By the assumption of the induction, for any $x \in X$,

$$
H \cap T(x) \supset\left(\bigcap_{i=3}^{n+1} F\left(x_{i} ; \bar{z}\right) \cap F(x ; \bar{z})\right) \neq \varnothing .
$$

It follows from condition (2) and (3), for $x_{1}, x_{2} \in X$ there exist $x_{1}^{\prime}, x_{2}^{\prime} \in \Gamma\left(x_{1}, x_{2}\right)$ such that $T\left(x_{i}^{\prime}\right) \subset T\left(x_{i}\right), i=1,2$ and $T(x) \subset \bigcup_{i=1}^{2} T\left(x_{i}\right)$ for all $X \in \Gamma\left(x_{1}, x_{2}\right)$. Hence we have

$$
H \cap T(x) \subset \bigcup_{i=1}^{2}\left(H \cap T\left(x_{i}\right)\right), \quad \forall x \in \Gamma\left(x_{1}, x_{2}\right) .
$$

By condition (3),

$$
\bigcap_{i=1}^{2} \mathrm{cl}\left(H \cap T\left(x_{i}\right)\right) \subset \bigcap_{i=1}^{n+1} F\left(x_{i}, z_{i}\right) \neq \varnothing .
$$

This shows that $H \cap T\left(x_{1}\right)$ and $H \cap T\left(x_{2}\right)$ are separated. Letting $I_{i}=\left\{x \in \Gamma\left(x_{1}, x_{2}\right)\right.$ : 
$\left.H \cap T(x) \subset H \cap T\left(x_{i}\right)\right\}, i=1$, 2, from (3.5) and $T\left(x_{i}^{\prime}\right) \subset T\left(x_{i}\right), i=1$, 2, we know that $x_{i}^{\prime} \in I_{i}, i=1,2$ and $I_{1} \cup I_{2}=\Gamma\left(x_{1}, x_{2}\right)$. Since $\Gamma\left(x_{1}, x_{2}\right)$ is connected, either $I_{1} \cap \operatorname{cl} I_{2}$ or $\operatorname{cl} I_{1} \cap I_{2}$ is nonempty. Without loss of generality, we can assume that $I_{1} \cup \operatorname{cl} I_{2} \neq \varnothing$. Taking $x_{0} \in I_{1} \cap \operatorname{cl} I_{2}$, then there exists a net $\left\{x_{\gamma}\right\}_{\gamma \in \theta} \subset I_{2}$ such that $x_{\gamma} \rightarrow x_{0}$ and $H \cap T\left(x_{1}\right) \supset H \cap T\left(x_{0}\right) \neq \varnothing$. Taking $y_{0} \in H \cap T\left(x_{0}\right)$, we have

$$
\varphi\left(x_{0}, y_{0}\right)<z_{1}
$$

It follows from (3.6) that $y_{0} \notin H \cap T\left(x_{2}\right)$. Hence, $y_{0} \notin H \cap T\left(x_{\gamma}\right)$ for all $\gamma \in \theta$, that is, $\varphi\left(x_{\gamma}, y_{0}\right) \geq z_{1}$ for all $\gamma \in \theta$.

By condition (2) and $x_{\gamma} \rightarrow x_{0}$, we have $\varphi\left(x_{0}, y_{0}\right) \geq z_{1}$. This contradicts (3.7). Therefore $\Omega$ has finite intersection property.

By condition (4), $K=\bigcap_{x \in A_{0}} F\left(x, z_{0}\right)$ is compact and $\{F(x, z) \cap K: x \in X$, $\left.z \in z_{\text {. }}\right\}$ has the finite intersection property, therefore we have $\bigcap_{x \in X, z \in z .} F(x, z)=$ $\bigcap_{x \in X, z \in z_{\bullet}}(F(x, z) \cap K) \neq \varnothing$, and so $\inf _{y \in Y} \sup _{x \in X} \varphi(x, y) \leq z_{\text {. }}$ This completes the proof.

REMARK 3.4. Theorem 3.3 improves and extends Theorem 1 in Lin and Quan [10].

COROLLARY 3.5. Let $X$ be a generalized interval space, $Y$ a topological space, $E$ a completely dense linear ordered space, and $f: X \times X \rightarrow E$ a function satisfying the following conditions:

(1) $f(x, y)$ is T-quasi-concave in $x$ and it is upper semi-continuous on any generalized interval of $X$;

(2) $f(x, y)$ is lower semi-continuous in $y$ and for any $A \in Д(X)$, the set

$$
\bigcap_{x \in A}\{y \in Y: f(x, y)<\alpha\} \in \mathscr{W}(Y), \quad \forall \alpha \in E
$$

(3) there exist an $A_{0} \in Д(X)$ and a $z_{0}>\sup _{x \in X} \inf _{y \in Y} f(x, y)$ such that

$$
\bigcap_{x \in A_{0}}\left\{y \in Y: f(x, y) \leq z_{0}\right\} \in \mathscr{W}(Y)
$$

then $\inf _{y \in Y} \sup _{x \in X} f(x, y)=\sup _{x \in X} \inf _{y \in Y} f(x, y)$.

Proof. It is obvious that inf $\operatorname{y\in Y}_{\mathcal{S u p}_{x \in X}} f(x, y) \geq \sup _{x \in X} \inf _{y \in Y} f(x, y)$. On the other hand, by Theorem 3.3 we know that $\inf _{y \in Y} \sup _{x \in X} f(x, y) \leq \sup _{x \in X} \inf _{y \in Y} f(x, y)$. This completes the proof.

REMARK 3.6. The corresponding results in Kindler and Trost [8], Tuy [7], Cheng and Lin [2], Brezis et al. [1], Komornik [9], Geraghty and Lin [4], Stacho [13], Sion [12], Wu [15], and Hartung [6] all are the special cases of Corollary 3.5.

COROLLARY 3.7. Let $X$ be a compact generalized interval space, E a completely dense linear ordered space, and $\varphi, \phi: X \times X \rightarrow E, \theta \in E$. If the following conditions are satisfied:

(1) for any $(x, y) \in X \times X, \varphi(x, y) \leq \phi(x, y)$;

(2) $\varphi(x, y)$ is T-quasi-concave in $x$ and it is upper semi-continuous on any generalized interval of $X ; \phi(x, y)$ is upper semi-continuous in $x$; 
(3) $\varphi(x, y)$ is lower semi-continuous in $y$, and for any $A \in Д(X)$ and any $\alpha \in E$, the set

$$
\bigcap_{x \in A}\{y \in X: f(x, y)<\alpha\} \in \mathscr{W}(X),
$$

then
(1) there exists $\bar{x} \in X$ such that $\phi(\bar{x}, \bar{x})>\theta$, or
(2) there exists $\bar{y} \in X$ such that $\varphi(x, \bar{y}) \leq \theta$ for all $x \in X$.

Proof. Suppose that (1) is not true, then for any $x \in X$ we have $\phi(x, x) \leq \theta$. By condition (2), $\inf _{y \in Y} \phi(x, y)$ is upper semi-continuous in $x$. Moreover, by Theorem 3.3 and the compactness of $X$ there exists an $\bar{x} \in X$ such that

$$
\inf _{y \in X} \sup _{x \in X} \varphi(x, y) \leq \sup _{x \in X} \inf _{y \in X} \phi(x, y)=\inf _{y \in X} \phi(\bar{x}, y) \leq \phi(\bar{x}, \bar{x}) \leq \theta .
$$

Since $X$ is compact, by condition (3) there exists a $\bar{y} \in X$ such that

$$
\varphi(x, \bar{y}) \leq \sup _{x \in X} \varphi(x, \bar{y})=\inf _{y \in X} \sup _{x \in X} \varphi(x, y), \quad \forall x \in X .
$$

COROLLARY 3.8. Let $X$ be a compact generalized interval space, $Y$ a compact topological space, E a completely dense linear ordered space, and $f: X \times Y \rightarrow E$ a mapping satisfying the following conditions:

(1) $x \mapsto f(x, y)$ is T-quasi-concave and upper semi-continuous;

(2) $y \mapsto f(x, y)$ is lower semi-continuous;

(3) for any $\alpha \in E$, any $A \in Д(X), \bigcap_{x \in A}\{y \in Y: f(x, y)<\alpha\} \in \nVdash(Y)$, then there exists a saddle point $(\bar{x}, \bar{y}) \in X \times Y$ such that $f(x, \bar{y}) \leq f(\bar{x}, \bar{y}) \leq f(\bar{x}, y)$ for all $x \in X$ and for all $y \in Y$.

Proof. If $\sup _{x \in X} \inf _{y \in Y} f(x, y)$ is a greatest element in $E$, then it is obvious that

$$
\inf _{y \in Y} \sup _{x \in X} f(x, y)=\sup _{x \in X} \inf _{y \in Y} f(x, y) .
$$

If $\sup _{x \in X} \inf _{y \in Y} f(x, y)$ is not a greatest element in $E$, then $f$ satisfies all the conditions in Corollary 3.5 obviously. By Corollary 3.5, we also have

$$
\inf _{y \in Y} \sup _{x \in X} f(x, y)=\sup _{x \in X} \inf _{y \in Y} f(x, y) .
$$

In view of the compactness of $X$ and $Y$, there exist $\bar{x} \in X$ and $\bar{y} \in Y$ such that

$$
\begin{aligned}
& \sup _{x \in X} \inf _{y \in Y} f(x, y)=\inf _{y \in Y} f(\bar{x}, y) \leq f(\bar{x}, y), \quad \forall y \in Y, \\
& \inf _{y \in Y} \sup _{x \in X} f(x, y)=\sup _{x \in X} f(x, \bar{y}) \geq f(x, \bar{y}), \quad \forall x \in X .
\end{aligned}
$$

Therefore, we have $f(\bar{x}, y) \geq f(x, \bar{y})$ for all $y \in Y$ and for all $x \in X$.

Taking $x=\bar{x}$ in the preceding inequality, we have $f(\bar{x}, y) \geq f(\bar{x}, \bar{y})$ for all $y \in Y$. Taking $y=\bar{y}$ in the preceding inequality, we have $f(\bar{x}, \bar{y}) \geq f(x, \bar{y})$ for all $x \in X$. Therefore, we have

$$
f(x, \bar{y}) \leq f(\bar{x}, \bar{y}) \leq f(\bar{x}, y), \quad \forall y \in Y, \forall x \in X .
$$


COROLlARY 3.9 (von Neumann). Let $C, F$ be two Hausdorff topological vector spaces, $X \subset C, Y \subset F$ two compact convex subsets, and $\phi: X \times Y \rightarrow \mathbb{R}$ a function satisfying the following conditions:

(1) for any $x \in X, \phi(x, y)$ is an upper semi-continuous concave function in $y$;

(2) for any $y \in Y, \phi(x, y)$ is a lower semi-continuous convex function in $x$, then there exists a saddle point $(\bar{x}, \bar{y}) \in X \times Y$ of $\varphi$.

Proof. For any $x_{1}, x_{2} \in X$ and for all $y_{1}, y_{2} \in Y$, letting

$$
\Gamma\left(x_{1}, x_{2}\right)=\operatorname{co}\left\{x_{1}, x_{2}\right\}, \quad \Gamma\left(y_{1}, y_{2}\right)=\operatorname{co}\left\{y_{1}, y_{2}\right\},
$$

then $\varphi$ satisfies all the conditions in Corollary 3.8. Therefore, the conclusion of Corollary 3.9 follows from Corollary 3.8 immediately.

COROLLARY 3.10. Let $X$ be a compact generalized interval space, $f: X \times X \rightarrow \mathbb{R}$, and $h: X \rightarrow \mathbb{R}$ two functions satisfying the following conditions:

(1) for any $x \in X, f(x, x) \leq 0$;

(2) $x \mapsto f(x, y)$ is upper semi-continuous and $y \mapsto f(x, y)$ is lower semi-continuous; $h$ is upper semi-continuous;

(3) $f(x, y)+h(x)$ is $T$-quasi-concave in $x$;

(4) for any $A \in Д(X)$ and for any $\alpha \in \mathbb{R}$ the set $\bigcap_{x \in A}\{y \in Y: f(x, y)-h(x)<\alpha\} \in$ $\Psi(X)$,

then there exists a $\bar{y} \in X$ such that $f(x, \bar{y}) \leq h(\bar{y})-h(x)$ for all $x \in X$.

Proof. Letting $g(x, y)=f(x, y)-h(y)+h(y)$, it is easy to prove that $g$ satisfies all the conditions in Corollary 3.8. Hence, there exist $x^{\bullet}, y^{\bullet} \in X$ such that $g\left(x, y^{\bullet}\right) \leq$ $g\left(x^{\bullet}, y\right)$ for all $x, y \in X$. Hence $g\left(x, y^{\bullet}\right) \leq g\left(x^{\bullet}, x^{\bullet}\right) \leq 0$ for all $x \in X$, that is, $f\left(x, y^{\bullet}\right) \leq h\left(y^{\bullet}\right)-h(x)$ for all $x \in X$.

THEOREM 3.11. Let $X$ be a generalized interval space, $Y$ a topological space, $E$ a completely dense linear ordered space, and $\phi, \varphi: X \times X \rightarrow E$ two mappings satisfying the following conditions:

(1) for any $(x, y) \in X \times Y, \varphi(x, y) \leq \phi(x, y)$;

(2) $x \mapsto \varphi(x, y)$ is T-quasi-concave and upper semi-continuous, $y \mapsto \varphi(x, y)$ is lower semi-continuous;

(3) $\phi(x, y)$ is upper semi-continuous in $x$;

(4) there exist a compact $K$ of $X$ and a nonempty subset $Y_{0}$ of $Y$ satisfying the following condition: for any $B \in Д(Y)$ there exists a compact set $M$ containing $Y_{0} \cup B$ such that for any $A \in Д(X)$ and for any $\alpha \in E$ the set $\bigcap_{x \in A}\{y \in M$ : $\varphi(x, y)<\alpha\} \in \mathscr{W}(Y)$, and

$$
\sup _{x \notin K} \inf _{y \in Y_{0}} \phi(x, y) \leq \sup _{x \in X} \inf _{y \in Y} \phi(x, y),
$$

then $\inf _{y \in Y} \sup _{x \in X} \varphi(x, y) \leq \sup _{x \in X} \inf _{y \in Y} \phi(x, y)$.

Proof. Letting $z^{\bullet}=\inf _{y \in Y} \sup _{x \in X} \varphi(x, y), z \cdot=\sup _{x \in X} \inf _{y \in Y} \varnothing(x, y)$. From the

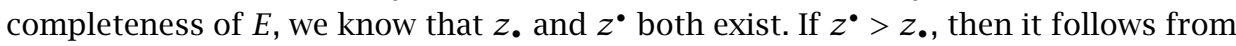
the density of $E$ that there exists a $\bar{z} \in E$ such that $z^{\bullet}>\bar{z}>z$. Let

$$
F(y)=\{x \in X: \phi(x, y) \geq \bar{z}\}, \quad y \in Y .
$$


By condition (3), $F(y)$ is closed for all $y \in Y$. From condition (4), for any $y_{1}, \ldots, y_{n} \in Y$ there exists a compact set $M$ containing $Y_{0} \bigcup\left\{y_{1}, \ldots, y_{n}\right\}$. It is easy to see that $\phi, \varphi$ : $X \times M \rightarrow E$ satisfy all the conditions in Theorem 3.3. By Theorem 3.3, we know

$$
\inf _{y \in M} \sup _{x \in X} \varphi(x, y) \leq \sup _{x \in X} \inf _{y \in M} \phi(x, y)
$$

On the other hand, since $\inf _{y \in M} \sup _{x \in X} \varphi(x, y) \geq \inf _{y \in Y} \sup _{x \in X} \varphi(x, y)=z^{\bullet}>\bar{z}$, $\sup _{x \in X} \inf _{y \in M} \phi(x, y)>\bar{z}$, and so $\bigcap_{y \in M} F(y) \neq \varnothing$. Hence we have $\bigcap_{y \in Y_{0}} F(y) \supset$ $\bigcap_{y \in M} F(y) \neq \varnothing$. By condition (4), we have $\sup _{x \notin K} \inf _{y \in Y_{0}} \phi(x, y) \leq z$. $<\bar{z}$. Hence, for all $x \in X \backslash K$ we have $\inf _{y \in Y_{0}} \phi(x, y) \leq z$. $<\bar{z}$, and so there exists a $y_{0} \in Y_{0}$ such that $\phi\left(x, y_{0}\right)<\bar{z}$, that is, $x \notin F\left(y_{0}\right)$. Hence $x \notin \bigcap_{y \in y_{0}} F(y)=D$. This implies that $D \subset K$. Since $K$ is compact and $D$ is closed, then $D$ is a compact set.

Summing up the above arguments, we have proved that $\{F(y) \cap D: y \in Y\}$ is a family of compact sets in $D$ having the finite intersection property. Hence, we have

$$
\bigcap_{y \in Y} F(y)=\bigcap_{y \in Y}(F(y) \bigcap D) \neq \varnothing .
$$

Therefore $z_{\bullet}=\sup _{x \in X} \inf _{y \in Y} \phi(x, y) \geq \bar{z}$. This contradicts the choice of $\bar{z}$. Therefore $z^{\bullet} \leq z_{\text {. }}$ This completes the proof.

REMARK 3.12. Theorem 3.11 improves the corresponding results in Ha [5] and Lin and Quan [10]. From Theorem 3.11 we can obtain the following results.

COROLLARY 3.13. Let $X$ be a generalized interval space, $Y$ a topological space, $E$ a completely dense linear ordered space, and $\varphi: X \times Y \rightarrow E$ a mapping satisfying the following conditions:

(1) $\varphi(x, y)$ is T-quasi-concave and upper semi-continuous in $x$ and it is lower semicontinuous in $y$;

(2) there exist a compact subset $K$ in $X$ and a nonempty subset $Y_{0}$ in $Y$ satisfying the following condition: for any $B \in Д(Y)$ there exists a compact set $M$ containing $Y_{0} \cup B$ such that for any $A \in Д(X)$ and for any $\alpha \in E, \bigcap_{x \in A}\{y \in M: \varphi(x, y)<$ $\alpha\} \in \mathscr{W}(Y)$ and

$$
\sup _{x \notin K} \inf _{y \in Y_{0}} \varphi(x, y) \leq \sup _{x \in X} \inf _{y \in Y} \varphi(x, y),
$$

then $\inf _{y \in Y} \sup _{x \in X} \varphi(x, y)=\sup _{x \in X} \inf _{y \in Y} \varphi(x, y)$.

TheOREM 3.14. Let $X$ be a generalized interval space, $Y$ a topological space, $E$ a completely dense linear ordered space, $\varphi: X \times Y \rightarrow E$ lower semi-continuous, and $\phi: X \times$ $Y \rightarrow E$ upper semi-continuous in $x$. Suppose that the following conditions are satisfied:

(1) $\varphi(x, y)$ is $T$-quasi-concave in $x$ and $\varphi(x, y) \leq \phi(x, y)$ for all $(x, y) \in X \times Y$;

(2) there exist a compact subset $K$ in $X$ and a nonempty subset $Y_{0}$ in $Y$ satisfying the following condition: for any $B \in Д(Y)$ there exists a compact set $M$ containing $Y_{0} \cup B$ such that for any $A \in Д(X)$ and for all $\alpha \in E, \bigcap_{x \in A}\{y \in M: \varphi(x, y) \leq$ $\alpha\} \in \mathscr{W}(Y)$ and

$$
\sup _{x \notin K} \inf _{y \in Y_{0}} \phi(x, y) \leq \sup _{x \in X} \inf _{y \in Y} \phi(x, y),
$$


then $\inf _{y \in Y} \sup _{x \in X} \varphi(x, y) \leq \sup _{x \in X} \inf _{y \in Y} \phi(x, y)$.

Proof. Letting $z^{\bullet}=\inf _{y \in Y} \sup _{x \in X}, \varphi(x, y), z \cdot=\sup _{x \in X} \inf _{y \in Y} \phi(x, y)$, if $z^{\bullet}>z_{\bullet}$, then by the density of $E$, there exists a $\bar{z} \in E$ such that $z^{\bullet}>\bar{z}>z_{\text {. }}$ Defining $F(y)=$ $\{x \in X: \phi(x, y) \geq \bar{z}\}, y \in Y$, since $x \mapsto \phi(x, y)$ is upper semi-continuous, we know that $F(y)$ is closed for all $y \in Y$. Defining $D=\bigcap_{y \in Y_{0}} F(y)$, then $D$ is closed.

On the other hand, for any finite set $\left\{y_{1}, \ldots, y_{n}\right\} \subset Y$, it follows from condition (2) that there exists a compact subset $M$ which contains $Y_{0} \bigcup\left\{y_{1}, \ldots, y_{n}\right\}$. Obviously, we can prove that $\varphi: X \times M \rightarrow E$ satisfies all the conditions in Theorem 3.1, we have

$$
\inf _{y \in M} \sup _{x \in X} \varphi(x, y)=\sup _{x \in X} \inf _{y \in M} \varphi(x, y)
$$

and so we have

$$
\begin{aligned}
\sup _{x \in X} \inf _{y \in M} \phi(x, y) & \geq \sup _{x \in X} \inf _{y \in M} \varphi(x, y)=\inf _{y \in M} \sup _{x \in X} \varphi(x, y) \\
& \geq \inf _{y \in Y} \sup _{x \in X} \varphi(x, y)=z^{\bullet}>\bar{z} .
\end{aligned}
$$

By the same method as in Theorem 3.11, we can prove $z_{\bullet} \geq \bar{z}$. This contradicts the choice of $\bar{z}$. Therefore we have $z^{\bullet} \leq z$. This completes the proof.

From Theorem 3.1 we can obtain the following theorem.

THEOREM 3.15. Let $X$ be a compact generalized interval space, E a completely dense linear ordered space, and $\varphi: X \times X \rightarrow E$ a lower semi-continuous function satisfying the following conditions:

(1) $x \mapsto \varphi(x, y)$ is $T$-quasi-concave;

(2) for any $A \in Д(X)$ and for any $\alpha \in E, \bigcap_{x \in A}\{y \in X: \varphi(x, y) \leq \alpha\} \in \mathscr{K}(X)$, then

(1) there exists an $\bar{x} \in X$ such that $\varphi(\bar{x}, \bar{x})>\theta$; or

(2) there exists an $\bar{y} \in X$ such that $\varphi(x, \bar{y}) \leq \theta$ for all $x \in X$.

Proof. Suppose the conclusion (1) is not true, then for any $x \in X$ we have $\varphi(x, x)$ $\leq \theta$. By Theorem 3.1 the following equation holds:

$$
\sup _{x \in X} \inf _{y \in X} \varphi(x, y)=\inf _{y \in X} \sup _{x \in X} \varphi(x, y) .
$$

Since for any $x \in X$ inf $_{y \in X} \varphi(x, y) \leq \varphi(x, x) \leq \theta$, we have $\sup _{x \in X} \inf _{y \in X} \varphi(x, y) \leq$ $\theta$, and so we have inf $\operatorname{in}_{y \in X} \sup _{x \in X} \varphi(x, y) \leq \theta$. By the compactness of $X$ and the lower semi-continuity of $\sup _{x \in X} \varphi(x, y)$ in $y$, there exists a $\bar{y} \in X$ such that

$$
\sup _{x \in X} \varphi(x, \bar{y})=\inf _{y \in X} \sup _{x \in X} \varphi(x, y) \leq \theta,
$$

that is, $\varphi(x, \bar{y}) \leq \theta$ for all $x \in X$. Therefore the conclusion (2) is true.

\section{Application to coincidence problem and fixed point problem}

THEOREM 4.1. Let $X$ be a compact generalized interval space, $Y$ a topological space, $F: X \rightarrow 2^{Y}$ a mapping with closed values, and $s \in C(X, Y)$ a given mapping. If the 
following conditions are satisfied:

(1) for any $x \in X, s^{-1} F(x) \neq \varnothing$ and $F^{-1}(s(x))$ is open;

(2) for any $A \in Д(X), X \backslash\left(\bigcup_{x \in A} F^{-1}(s(x))\right) \in \mathscr{W}(X)$;

(3) for any $x_{1}, x_{2} \in X$ and for any $y \in \bigcap_{i=1}^{2} F^{-1}\left(s\left(x_{i}\right)\right), s\left(\Gamma\left(x_{1}, x_{2}\right)\right) \subset F(y)$ there exist $x_{1}^{\prime}, x_{2}^{\prime} \in \Gamma\left(x_{1}, x_{2}\right)$ such that $F^{-1}\left(s\left(x_{i}\right)\right) \subset F^{-1}\left(s\left(x_{i}^{\prime}\right)\right), i=1,2$,

then there exists an $\bar{x} \in X$ such that $s(\bar{x}) \in F(\bar{x})$.

Proof. For any $(x, z) \in X \times X$, let

$$
f(x, z)= \begin{cases}0, & s(x) \notin F(x), \\ 1, & s(x) \in F(x) .\end{cases}
$$

If the conclusion is not true, then for any $x \in X$ we have $f(x, x)=0$.

Since for any $x \in X$ and for any $r \in \mathbb{R}$,

$$
\{z \in X: f(x, z) \leq r\}= \begin{cases}\varnothing, & r<0, \\ F^{\bullet}(s(x)), & 0 \leq r<1, \\ X, & r \geq 1,\end{cases}
$$

where $F^{\bullet}(x)=X \mid F^{-1}(x)$, it follows from conditions (1) and (2) that $f(x, z)$ is lower semi-continuous in $z$ and for any $A \in Д(X)$ and for any $r \in \mathbb{R}, \bigcap_{x \in A}\{z \in X: f(x, z)<$ $r\} \in \mathscr{W}(X)$. On the other hand, since for any $z \in X$ and for any $r \in \mathbb{R}$,

$$
\{x \in X: f(x, z) \geq r\}= \begin{cases}X, & r \leq 0, \\ s^{-1}(F(z)), & 0<r \leq 1, \\ \varnothing, & r>1\end{cases}
$$

and $F$ is a mapping with closed values, $f(x, z)$ is upper semi-continuous in $x$.

Letting $G(x)=F^{\bullet}(s(x))$, then $G$ is a mapping from $X$ into $2^{X}$ and for any $(x, z) \in$ $X \times X$,

$$
f(x, z)= \begin{cases}0, & z \in G(x), \\ 1, & z \notin G(x) .\end{cases}
$$

Next we prove that $G$ is a $T$-KKM mapping. In fact, from condition (3) for any $x_{1}, x_{2} \in$ $X$, we have $s\left(\Gamma\left(x_{1}, x_{2}\right)\right) \subset F(z)$ for all $z \in \bigcap_{i=1}^{2} F^{-1}\left(s\left(x_{i}\right)\right)$. Hence for any $x \in \Gamma\left(x_{1}, x_{2}\right)$, and for any $z \in \bigcap_{i=1}^{2} F^{-1}\left(s\left(x_{i}\right)\right), s(x) \in F(z)$.

Therefore, for all $x \in \Gamma\left(x_{1}, x_{2}\right)$ and for any $z \in \bigcap_{i=1}^{2} F^{-1}\left(s\left(x_{i}\right)\right)$, we have $z \in$ $F^{-1}(s(x))$. This implies that $\bigcap_{i=1}^{2} F^{-1}\left(s\left(x_{i}\right)\right) \subset F^{-1}(s(x))$ for all $x \in \Gamma\left(x_{1}, x_{2}\right)$. Therefore, $G(x) \subset \bigcup_{i=1}^{2} G\left(x_{i}\right)$ for all $x \in \Gamma\left(x_{1}, x_{2}\right)$. Again by condition (3) there exist $x_{1}^{\prime}, x_{2}^{\prime} \in$ $\Gamma\left(x_{1}, x_{2}\right)$ such that $F^{-1}\left(s\left(x_{i}\right)\right) \subset F^{-1}\left(s\left(x_{i}^{\prime}\right)\right), i=1,2$. Thus $G\left(x_{i}\right) \subset G\left(x_{i}^{\prime}\right), i=1,2$. This shows that $G$ is a $T$-KKM mapping. By Lemma 1.8 it is easy to prove that $f(x, y)$ is a $T$-quasi-concave mapping in $x$. Since $X$ is compact, $f$ satisfies all the conditions in Corollary 3.7. By Corollary 3.7 there exists a $\bar{z} \in X$ such that $f(x, \bar{z}) \leq 0$ for all $x \in X$, and so $f(x, \bar{z})=0$ for all $x \in X$, that is, $s(x) \notin F(\bar{z})$ for all $x \in X$. Hence $x \notin s^{-1} F(\bar{z})$ for all $x \in X$, and so $s^{-1} F(\bar{z})=\varnothing$.

This contradicts condition (1). This completes the proof. 
From Theorem 4.1 we obtain the following fixed point theorem.

COROLLARY 4.2. Let $X$ be a generalized interval space, $C$ be a compact $T$-convex subset of $X$, and $F: C \rightarrow 2^{C}$ be a set-valued mapping with nonempty closed values. If the following conditions are satisfied:

(1) for any $x \in C, F^{-1}(x)$ is open in $C$;

(2) for any $A \in Д(C), C \backslash\left(\bigcup_{x \in A} F^{-1}(x)\right) \in \mathcal{W}(C)$;

(3) for any $x_{1}, x_{2} \in X, \Gamma\left(x_{1}, x_{2}\right) \subset F(y)$ for all $y \in \bigcap_{i=1}^{2} F^{-1}\left(x_{i}\right)$, and there exist $x_{1}^{\prime}, x_{2}^{\prime} \in \Gamma\left(x_{1}, x_{2}\right)$ such that $F^{-1}\left(x_{i}\right) \subset F^{-1}\left(x_{i}^{\prime}\right), i=1,2$,

then there exists an $\bar{x} \in C$ such that $\bar{x} \in F(\bar{x})$.

THEOREM 4.3. Let $X$ be a generalized interval space, $Y$ a topological space, $F: X \rightarrow 2^{Y}$ and $s \in C(X, Y)$ two mappings satisfying the following conditions:

(1) for any $x \in X, F^{-1}(s(x))$ is nonempty closed and $X \backslash s^{-1}(F(x))$ is T-convex;

(2) for any $A \in Д(X), \bigcap_{x \in A} s^{-1}(F(x)) \in \mathscr{W}(X)$;

(3) $F^{-1}: Y \rightarrow 2^{X}$ is upper semi-continuous and for any $x_{1}, x_{2} \in X$, there exist $x_{1}^{\prime}, x_{2}^{\prime} \in$ $\Gamma\left(x_{1}, x_{2}\right)$ such that $F^{-1}\left(s\left(x_{i}^{\prime}\right)\right) \subset F^{-1}\left(s\left(x_{i}\right)\right), i=1,2$;

(4) there exists an $A_{0} \in Д(X)$ such that $\bigcap_{x \in A_{0}} F^{-1}(s(x))$ is compact, then there exists an $\bar{x} \in X$ such that $s(\bar{x}) \in F(\bar{x})$.

Proof. Letting $G(x)=F^{-1}(s(x))$, then $G: X \rightarrow 2^{X}$ is a nonempty mapping and closed valued and $G$ satisfies condition (2) in Theorem 2.1 .

On the other hand, for any $x \in X$, let $W$ be an open set containing $G(x)$. Since $F^{-1}$ is upper semi-continuous, there exists an open neighborhood $V$ of $s(x)$ such that for any $y \in V$, we have $F^{-1}(y) \subset W$. Since $s \in C(x, y)$, there exists an open neighborhood $U$ of $x$ such that for any $z \in U$ we have $s(z) \in V$, and so for $z \in U$, we have $G(z)=F^{-1}(s(z)) \subset W$. This shows $G$ is an upper semi-continuous mapping.

Next we prove that $G: X \rightarrow 2^{X}$ is a $T$-KKM mapping. In fact, for any $x_{1}, x_{2} \in X$ and for any $x \in \Gamma\left(x_{1}, x_{2}\right)$, if $z \notin \bigcap_{i=1}^{2} G\left(x_{i}\right)$, then $z \notin F^{-1}\left(s\left(x_{i}\right)\right), i=1,2$, that is, $s\left(x_{i}\right) \notin F(z), i=1,2$, and so $\left\{x_{1}, x_{2}\right\} \subset X \backslash s^{-1} F(z)$. By the assumption that $X \backslash s^{-1} F(z)$ is $T$-convex, hence $\Gamma\left(x_{1}, x_{2}\right) \subset X \backslash s^{-1} F(z)$, and so $z \notin F^{-1}(s(x))$ for all $x \in \Gamma\left(x_{1}, x_{2}\right)$. This implies that

$$
G(x)=F^{-1}(s(x)) \subset \bigcup_{i=1}^{2} G\left(x_{i}\right) .
$$

Again by condition (3), there exist $x_{1}^{\prime}, x_{2}^{\prime} \in \Gamma\left(x_{1}, x_{2}\right)$ such that $G\left(x_{i}^{\prime}\right) \subset G\left(x_{i}\right), i=$ 1,2 . This means that $G$ is a $T$-KKM mapping.

In view of condition (4), we know that $G$ satisfies all the conditions in Theorem 2.1. By Theorem $2.1 \bigcap_{x \in X} G(x) \neq \varnothing$, and so there exists an $\bar{x} \in X$ such that $\bar{x} \in G(x)$ for all $x \in X$. Hence $\bar{x} \in G(\bar{x})$, that is, $\bar{x} \in F^{-1}(s(\bar{x}))$, and so $s(\bar{x}) \in F(\bar{x})$.

THEOREM 4.4. Let $K$ be a generalized interval space, $F: X \rightarrow 2^{X}$ a mapping with nonempty values. If $X$ is normal and the following conditions are satisfied:

(1) $F$ is transfer closed valued;

(2) $F$ is almost upper semi-continuous, that is, for any $x \in X$ and for any open set $V$ containing $F(x)$, there exists an open neighborhood $U$ of $x$ such that $F(y) \subset \operatorname{cl} V$ for all $y \in U$;

(3) for any $A \in Д(X), \bigcap_{x \in A} \operatorname{cl} F(x) \in \nVdash(X)$; 
(4) there exists an $A_{0} \in D(X)$ such that $\bigcap_{x \in A_{0}} \operatorname{cl} F(x)$ is compact;

(5) for any $x \in X, X \backslash F^{-1}(x)=F^{\bullet}(x)$ is $T$-convex, then $F$ has a fixed point in $X$.

Proof. For any $x \in X$, if $W$ is any open set containing $\operatorname{cl} F(x)$, by the normality of $X$, there exists an open set $V$ containing $\operatorname{cl} F(x)$ such that $\operatorname{cl} V \subset W$. It follows form the almost upper semi-continuity of $F$ that there exists an open neighborhood $U$ of $x$ such that $F(y) \subset \operatorname{cl} V$ for all $Y \in U$. Hence $\operatorname{cl} F(y) \subset \operatorname{cl} V \subset W$. This means that $F$ is upper semi-continuous.

Next we prove that $F$ is a $T$-KKM mapping. In fact, for any $x_{1}, x_{2} \in X$ if $X \notin$ $\bigcup_{i=1}^{2} F\left(x_{i}\right)$, then $x_{1}, x_{2} \notin F^{-1}(y)$. Since $F^{\bullet}(y)$ is $T$-convex, $\Gamma\left(x_{1}, x_{2}\right) \subset F^{\bullet}(y)$, and so for any $x \notin \Gamma\left(x_{1}, x_{2}\right)$ we have $x \notin F^{-1}(y)$, that is, $y \notin F(x)$. Therefore, $F(x) \subset$ $\bigcup_{i=1}^{2} F\left(x_{i}\right)$ for all $x \in \Gamma\left(x_{1}, x_{2}\right)$, and so $\operatorname{cl} F(x) \subset \bigcup_{i=1}^{2} \operatorname{cl} F\left(x_{i}\right)$ for all $x \in \Gamma\left(x_{1}, x_{2}\right)$. In view of condition (5) we know that $F$ is a $T$-KKM mapping.

Moreover, it is easy to prove that all conditions in Theorem 2.2 are satisfied. By Theorem 2.2, $\bigcap_{x \in X} F(x) \neq \varnothing$. Hence there exists an $\bar{x} \in X$ such that $\bar{x} \in F(x)$ for all $x \in X$, and so we have $\bar{x} \in F(\bar{x})$. This completes the proof.

\section{Application to economy equilibrium problem}

THEOREM 5.1. Let $X$ be a generalized interval space, $A, B, P: X \rightarrow 2^{X}$. If the following conditions are satisfied:

(1) for any $x \in X, A(x) \subset B(x), x \notin P(x), A(x)$, and $P(x)$ are nonempty closed sets;

(2) $A$ and $P$ are upper semi-continuous;

(3) for any $y \in X, A^{\bullet}(y) \cup P^{\bullet}(y)$ and $A^{\bullet}(y) \bigcup\{x \in X: x \in \operatorname{cl} B(x)\}$ are $T$-convex sets;

(4) for any $M, N \in Д(X),\left(\bigcap_{x \in M} A(x)\right) \cap\left(\bigcap_{x \in N} P(x)\right) \in \mathscr{X}(X)$;

(5) there exists $M_{0} \in Д(X)$ such that $\bigcap_{x \in M_{0}} A(x)$ is compact,

then $(X, A, B, P)$ has a Shafer-Sonneinschein equilibrium, that is, there exists an $\bar{x} \in X$ such that $\bar{x} \in \operatorname{cl} B(\bar{x})$ and $A(\bar{x}) \cap P(\bar{x}) \neq \varnothing$.

Proof. Suppose the contrary, for any $x \in X$ we have $x \notin \operatorname{cl} B(x)$ or $A(x) \cap P(x)=\varnothing$. Letting

$$
\begin{gathered}
D=\{x \in X: x \in \operatorname{cl} B(x)\}, \\
F(x)= \begin{cases}A(x) \cap P(x), & x \in D, \\
A(x), & x \notin D .\end{cases}
\end{gathered}
$$

By condition (1) and (2), $F$ is an upper semi-continuous mapping with nonempty closed values. For any $y \in X$,

$$
\begin{aligned}
F^{-1}(y) & =\{x \in X: y \in F(x)\} \\
& =\left\{x \in X: x \in A^{-1}(y) \cap P^{-1}(y)\right\} \cup\left\{x \notin D: x \in A^{-1}(y)\right\}, \\
F^{\bullet}(y) & =\left\{x \in X: x \in A^{\bullet}(y) \cup P^{\bullet}(y)\right\} \cap\left\{x \in X: x \in A^{\bullet}(y) \cup D\right\} \\
& =\left(A^{\bullet}(y) \cup P^{\bullet}(y)\right) \cap\left(A^{\bullet}(y) \cup D\right) .
\end{aligned}
$$


By condition (3), $F^{\bullet}(y)$ is a $T$-convex set; and by condition (4), $\bigcap_{x \in M} F(x) \in \mathscr{W}(X)$. It is obvious that $F$ satisfies all conditions in Theorem 2.1. By Theorem 2.1, there exists a $\bar{y} \in X$ such that $\bar{y} \in F(\bar{y})$. If $\bar{y} \notin D$, then $\bar{y} \in A(\bar{y}) \subset B(\bar{y})$ which is impossible; if $\bar{y} \in D$, then $\bar{y} \in P(\bar{y})$ which contradicts condition (1). Therefore, there exists an $\bar{x} \in X$ such that $\bar{x} \in \operatorname{cl} B(\bar{x})$ and $A(\bar{x}) \cap P(\bar{x}) \neq \varnothing$.

\section{REFERENCES}

[1] H. Brézis, L. Nirenberg, and G. Stampacchia, A remark on Ky Fan's minimax principle, Boll. Un. Mat. Ital. (4) 6 (1972), 293-300. MR 48\#2850. Zbl 264.49013.

[2] C. Z. Cheng and Y. H. Lin, A generalization of minimax theorems of fixed-point type, Acta Math. Sinica 34 (1991), no. 4, 502-507. MR 93c:49008. Zbl 0833.49008.

[3] K. Fan, Fixed-point and minimax theorems in locally convex topological linear spaces, Proc. Nat. Acad. Sci. U.S.A. 38 (1952), 121-126. MR 13,858d. Zbl 0047.35103.

[4] M. A. Geraghty and B.-L. Lin, Topological minimax theorems, Proc. Amer. Math. Soc. 91 (1984), no. 3, 377-380. MR 86c:49009. Zbl 533.90095.

[5] C. W. Ha, A noncompact minimax theorem, Pacific J. Math. 97 (1981), no. 1, 115-117. MR 83g:49024. Zbl 474.49015.

[6] J. Hartung, An extension of Sion's minimax theorem with an application to a method for constrained games, Pacific J. Math. 103 (1982), no. 2, 401-408. MR 84k:90101. Zbl 534.49009.

[7] T. Hoang, On a general minimax theorem, Soviet Math. Dokl. 15 (1974), 1689-1693 (Russian). Zbl 314.90088.

[8] J. Kindler and R. Trost, Minimax theorems for interval spaces, Acta Math. Hungar. 54 (1989), no. 1-2, 39-49. MR 91f:49009. Zbl 688.90061.

[9] V. Komornik, Minimax theorems for upper semicontinuous functions, Acta Math. Acad. Sci. Hungar. 40 (1982), no. 1-2, 159-163. MR 84h:49023. Zbl 505.49007.

[10] B.-L. Lin and X. C. Quan, A noncompact topological minimax theorem, J. Math. Anal. Appl. 161 (1991), no. 2, 587-590. MR 92i:49011. Zbl 779.49009.

[11]__ A two functions nonlinear minimax theorem, Fixed Point Theory and Applications (Marseille, 1989), Pitman Res. Notes Math. Ser., vol. 252, Longman Scientific \& Technical, Harlow, 1991, pp. 321-325. MR 92j:54023. Zbl 771.49003.

[12] M. Sion, On general minimax theorems, Pacific J. Math. 8 (1958), 171-176. MR 20\#3506. Zbl 081.11502.

[13] L. L. Stachó, Minimax theorems beyond topological vector spaces, Acta Sci. Math. (Szeged) 42 (1980), no. 1-2, 157-164. MR 82a:49017. Zbl 436.49017.

[14] G. Q. Tian, Generalizations of the FKKM theorem and the Ky Fan minimax inequality, with applications to maximal elements, price equilibrium, and complementarity, J. Math. Anal. Appl. 170 (1992), no. 2, 457-471. MR 94b:90014. Zbl 767.49007.

[15] W.-C. Wu, A remark on the fundamental theorem in the theory of games, Sci. Record (N.S.) 3 (1959), 229-233. MR 22\#13311.

Da-Cheng Wang: Department of Computers, Chongqing JiaOtong Institute, CHONGQING 400074, CHINA 


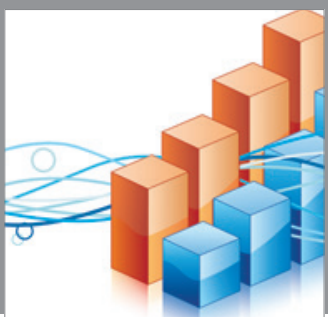

Advances in

Operations Research

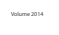

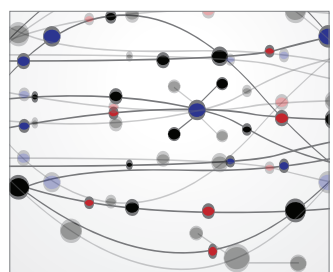

\section{The Scientific} World Journal
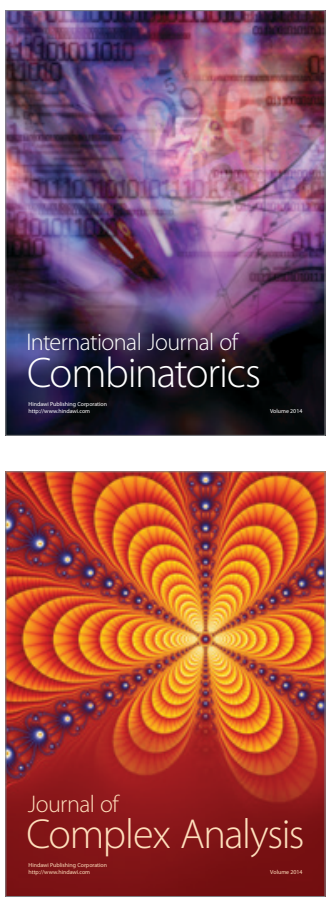

International Journal of

Mathematics and

Mathematical

Sciences
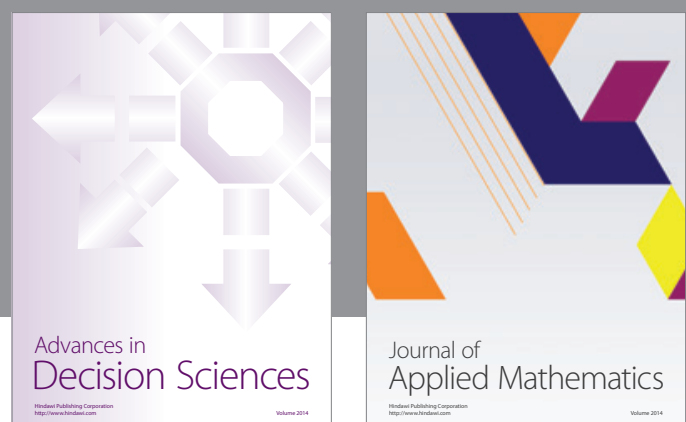

Journal of

Applied Mathematics
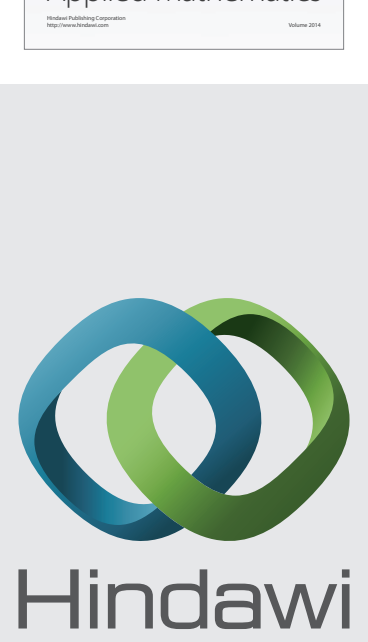

Submit your manuscripts at http://www.hindawi.com
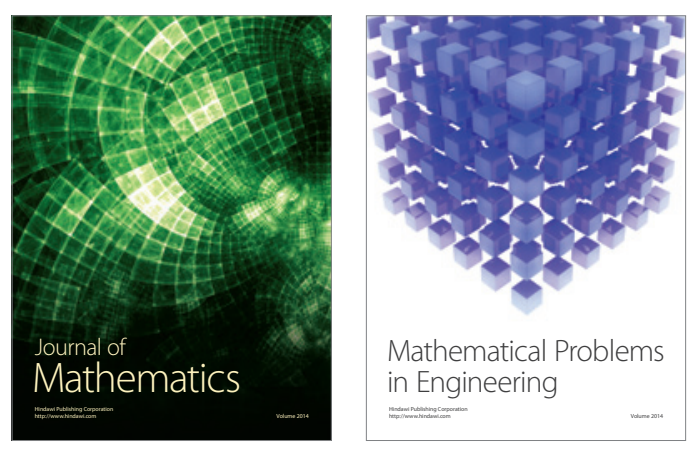

Mathematical Problems in Engineering
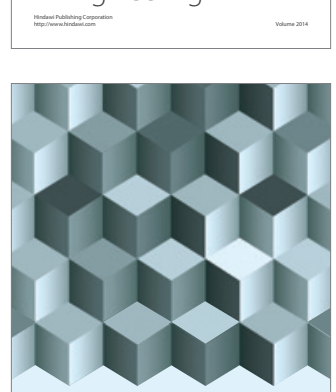

Journal of

Function Spaces
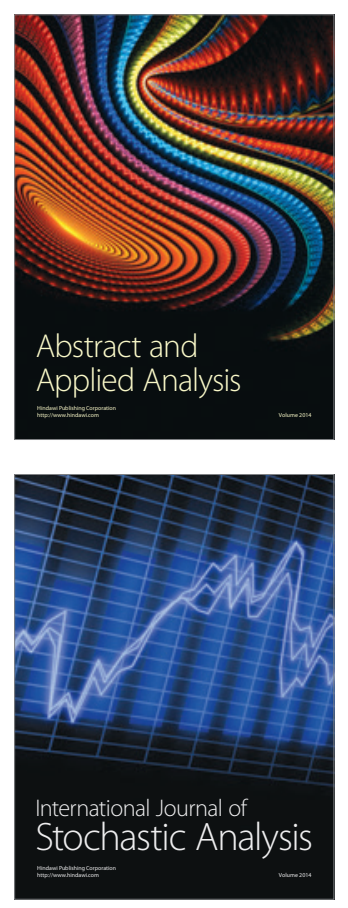

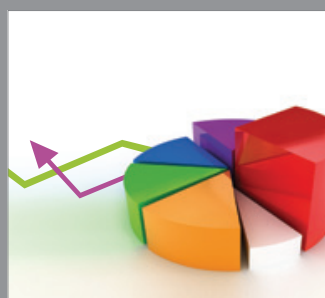

ournal of

Probability and Statistics

Promensencen
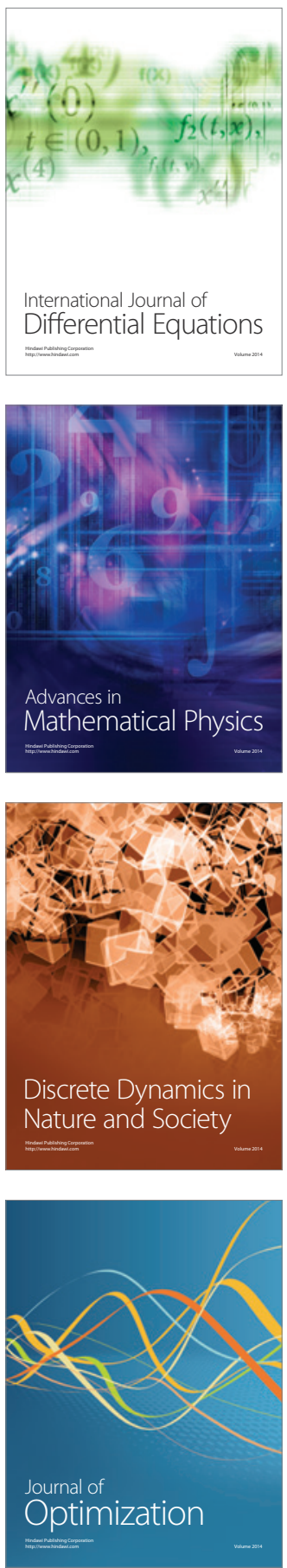